\title{
The Design and Application of Intelligent Network Cabling System for Building
}

\author{
Hong Mao \\ Chongqing Vocational Institute of Engineering, Jiangjin 402260, China \\ maoh@163.com
}

Keywords: Intelligent network for building, information application, network cabling.

\begin{abstract}
The development of Internet has changed people's life, science and technology promote the economic development. In modern building network cabling system, whole buildings or buildings at first were mainly reflected the comprehensive cabling. Integrated cabling system is mainly used for building in network transmission, it combines voice with image integrated together, and communicate with the outside world network system. The cabling system has shown many characteristics, such as expansibility, openness and flexibility, and independence. This article introduces the network cabling system composition and design, and points out what we should pay attention to in the concrete implementation of the problem.
\end{abstract}

\section{Introduction}

As the growing terminal equipment and the increasing complexity of the physical layer connection in information network, the challenges and costs associated with the management and maintenance of network physical layer is becoming surprising. [1] Traditional cabling system can't meet the various requirements of the growing demand. A new solution of intelligent cabling system should be shipped out. The intelligent network cabling system for building is an inevitable course with the development of information technology. [2] According to the request of daily life for people, the thesis analyzes the function architecture for the intelligent network cabling system for building, designs a home control center based on the technology of embedded web, defines the integrated communication criterion for the information appliance, develops the communication between the SANYO ECJ-218M electronic rice cooker and the control center, and achieves the remote control function to the appliance through the intelligent network cabling system. [3] With the development of science, technique and society economy, especially the coming of information age, there are more desires on building which offers an elegant, comfortable, convenient, secure and informational environment. So a series of baptism to traditional Structure Cabling System (SCS) is brought forward. Traditional design method that considers office, safety, fire control, communication respectively can't meet with the desires of modern consumers to the building. [4] Generic Cabling System (GCS) considers every cabling system synthetically, which can exert the function of building to tiptop and can satisfy consumers' demands. So it has been becoming the direction of the cabling technology.

\section{The technical route}

According to the actual demand for the application, the intelligent network cabling system is divided into four different functional parts, which are the environment maintenance subsystem, the security management subsystem, the information appliance subsystem and the automatic reading meter subsystem. The control center is carried out based on the ARM CPU, and takes on the home gateway function. The thesis achieves the configuration and installation of the uClinux, and based on which, develops the CGI and communication programs. The CGI programs response to the client request, and invoked by the web server Boa which is appropriative for the embedded OS. The criterion on the communication of the IA includes an up layer of application service and a low layer of communication control. [5] The application service layer achieves the function of device identification, parameters update, and the remote control to the devices. The communication control 
layer takes the responsibility of the address management, the priority management and the transfer check work. And the criterion is applied on the communication between the sanyo ECJ-218M electronic rice cooker and the control center. The thesis takes the sanyo ECJ-218M electronic rice cooker as application object, implements the serial communication interface connected to the control center by using the I/O ports and timer interrupt of the MCU of the cooker controller. The functional structure of the intelligent network cabling for building is shown in Fig. 1.

'FD
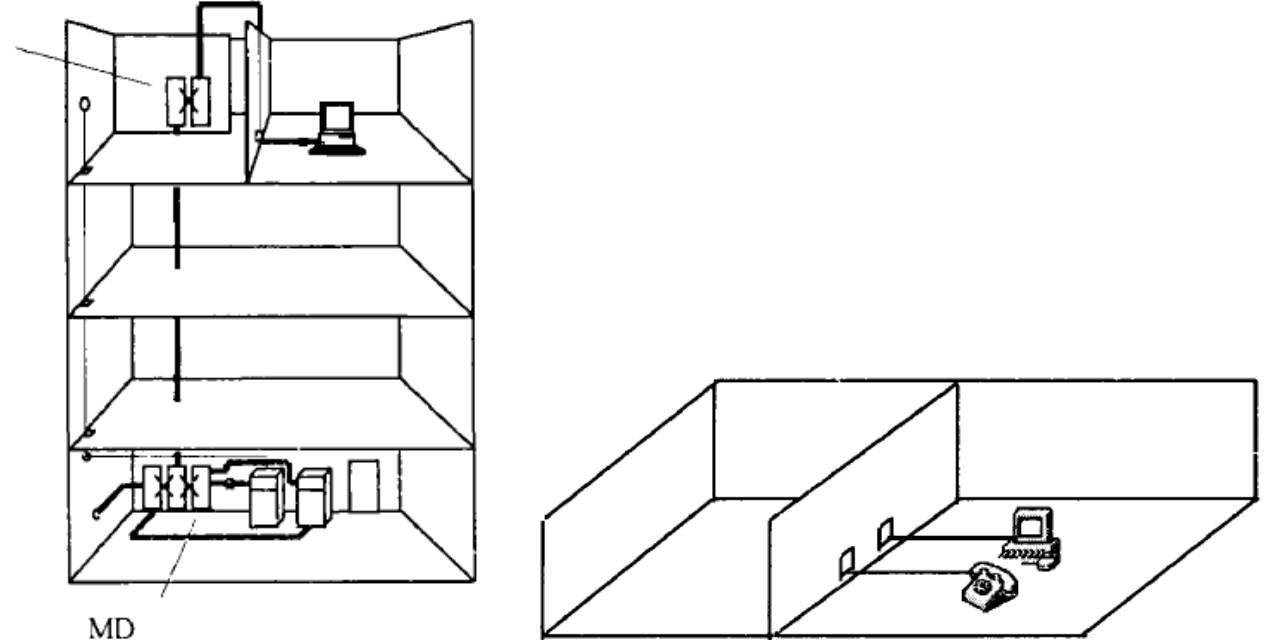

Fig. 1 The functional structure of the intelligent network cabling for building

\section{Method}

This paper first describes the traditional structured cabling systems become inefficient in the face of the growing size of the network, a new type of intelligent cabling system required by user need. Then, combined with a project examples, from the requirement of users, analysis the low efficiency and implementation for traditional cabling system. Prefer to the need for intelligent cabling system. Then introduced three kind of techniques for intelligent cabling systems, and analyze their principles, advantages and disadvantages. Select the appropriate technology solutions according to the needs of users. The circuit diagram of the serial communication is shown in Fig. 2,

After that, propose the design ideas of the various subsystem according to the requirements of intelligent cabling system. On intelligent patch panel mode, select dual patch panel mode as the implementation of the system solution after analysis and comparing the advantages and disadvantages of two different modes.

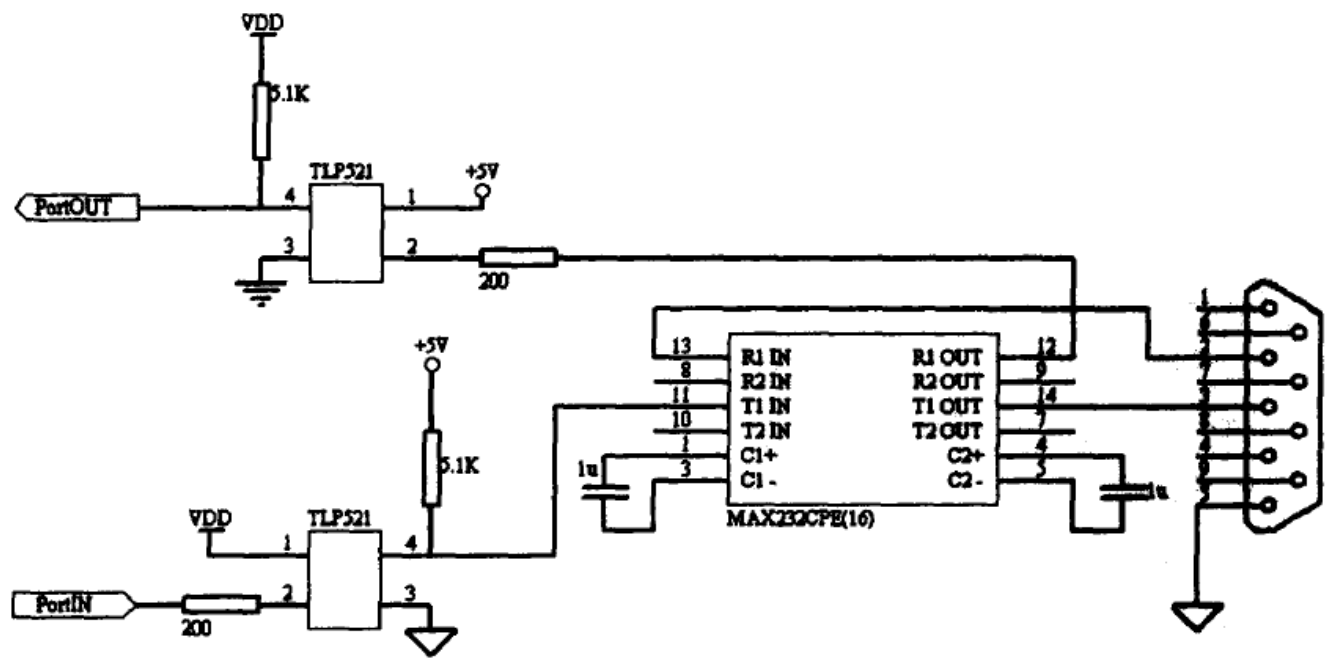

Fig. 2 The circuit diagram of the serial communication

Then, research intelligent cabling system how to monitor the physical layer port base on the use of structured cabling system and a dedicated jumper and smart patch panels. This paper also innovated propose the combined link and port technology for patch panel detect model, and applied the 
top-of-rack cabling solution to eh dual patch panel mode, which improve the efficiency of operation and reduce the overall costs. The research helps to better application of intelligent cabling system in the implementation process, and demonstrate its advantages and development direction of the current and future market.

The structure cabling system is the modern communication domain high tech crystallization, it has provided the most reasonable cabling way for the user, and depends upon its high quality the material, as soon as changes the traditional cabling appearance, could become the wisdom for the modernized building to lay the line foundation truly.

\section{Software design about cabling system of intelligent building}

It is a very imperious mission to develop a kind of design software, with which people can save time and lessen loads when confronting the development of Intelligent Building and GCS. The project of "Judgment System of Intelligent Building" put forward by the Science and Technology Hall of Hubei was confirmed to a strategic one in 2000 year, whose intention is to develop a suit of design software about Generic Cabling System of Intelligent Building. The thesis spreads through this task and the main contents are as following:

1 Embody the advantages of GCS through comparing GCS with SCS. It confirms the general design about Generic Cabling System of Intelligent building through analyzing and researching on GCS.

2. Study the application of Object-Oriented Technology (00T) on developing software. OOT is a very important study method even a key to develop the design software about GCS.

3. Analyze the design software about GCS in detail. The development of the design software about GCS utilizes Object-Oriented Analysis (00A) to abstract 19 objects from the things in the problem space. The 19 objects' abstraction and description is the important base of the design software about GCS.

4. Devise the design software about GCS by Object-Oriented Design (00D). This thesis transforms the 19 objects and their relations abstracted from OOA into the classes and relations in the problem space, and describe the classes in detail.

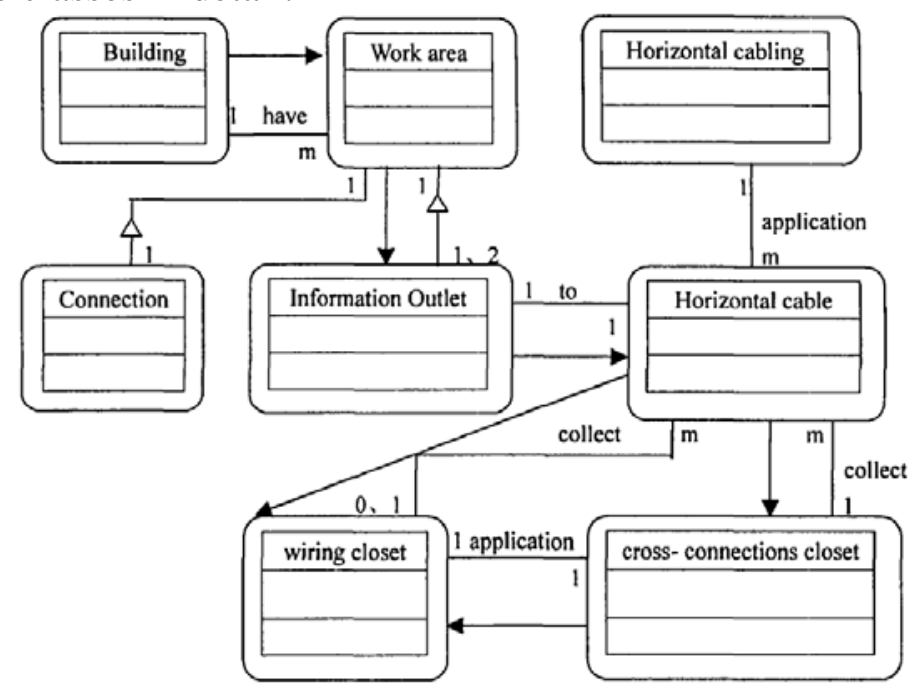

Fig. 3 The problem space of integrated cabling design software

The development of design software about GCS is necessary to the construction trade. This thesis describes the course of analysis and design of the design software about GCS by using OOT, which is the key to realize the design software, and is the indispensable base to develop software.

With the continually increasing demands from Intelligent Building Integrated, Cabling System are required to work with building automation systems, security systems more closely combined. As the most basic and important part of an intelligent building, Building automation system is a highly automated and integrated management and control systems by use of computers and network technology, automatic control technology and commutations technology. A variety of devices inside 
the building cam be connected to a control network through BAS; be unified control, These devices include air-conditioning, lighting, elevators, fire and security and so on. It ensures that the building could be comfortable and safe, achieving energy efficient. The problem space of integrated cabling design software is shown in Fig. 3. The following system test results are followed as Table 1.

Table 1 Test results

\begin{tabular}{ccccc}
\hline \multicolumn{5}{c}{ MAX ATTENUATION } \\
\hline \multicolumn{5}{c}{ Measuring at 20 ${ }^{\circ} \mathrm{C}$} \\
\hline Channel(100m) & \multicolumn{4}{c}{ Basic Link(94m) } \\
\hline F(Mhz) & Cat.4 & Cat.5 & Cat.4 & Cat.5 \\
\hline 1 & 2.6 & 2.5 & 2.2 & 2.1 \\
4 & 4.8 & 4.5 & 4.3 & 4 \\
8 & 6.7 & 6.3 & 6.8 & 5.7 \\
10 & 7.5 & 7 & 8.8 & 6.3 \\
16 & 9.9 & 9.2 & 9.9 & 8.2 \\
20 & 11 & 10.3 & & 9.2 \\
25 & & 11.4 & & 10.3 \\
31.25 & & 12.8 & 11.5 \\
62.5 & & 18.5 & 21.6 \\
100 & & 24 & & \\
\hline
\end{tabular}

The design of comprehensive intellectual building cabling system include various system circuit such as phonetic exchange system, data communications network, closed road monitoring systems, building visual interphone system, keep watching system, intellectual parking and public broadcasting system, to establish a real integration system which control by computer system. Those provide an environment of space which investment reasonable, high efficiency, elegant, comfortable, convenient, high safe for building.

\section{Conclusions}

This paper based on the practical demand, the thesis studies on the function architecture for the intelligent network cabling system for building. The thesis takes the SANYO 218MS electronic rice cooker as the object, carries out the network cabling function for the information appliance, achieves the remote control, error alarm and other functions, which is valuable to the development of both information appliance and building network cabling.

\section{References}

[1] John Park, Steve Mackay, Edwin Wright, Cabling basics Practical Data Communications for Instrumentation and Control, 2013: 112-123

[2] Goran Simic, Vladan Devedzic Building an intelligent system using modern Internet technologies Expert Systems with Applications, 2013, 25(2): 231-246.

[3] Hanif D. Sherali, Youngho Lee, Taehyung Park New modeling approaches for the design of local access transport area networks European Journal of Operational Research, 2010, 127(1): 94-108.

[4] Gilbert Held, The Physical Layer Cables Connectors Plugs and Jacks Understanding Data Communications From Fundamentals to Networking, 2011, 5: 183-234.

[5] LA Neminath Hubballi, Santosh Biswas, S. Roopa, Ritesh Ratti, Sukumar Nandi attack detection using Discrete Event Systems ISA Transactions, 2011, 50(1): 119-130. 